\title{
First-principles simulations of copper diffusion in tantalum and tantalum nitride
}

\author{
Yi Zhao and Gang Lu \\ Department of Physics and Astronomy, California State University Northridge, Northridge, California 91330-8268, USA
}

(Received 15 January 2009; revised manuscript received 16 March 2009; published 3 June 2009)

\begin{abstract}
To explore potential applications of tantalum and tantalum nitrides ( $\mathrm{TaN})$ as diffusion barrier materials in integrated circuits with $\mathrm{Cu}$ interconnects, we carry out detailed first-principles simulations of $\mathrm{Cu} / \mathrm{Ta}$ and $\mathrm{Cu} / \mathrm{TaN}$ systems. Various interfacial structures between $\mathrm{Cu}$ - and Ta-based compounds are examined by considering different surface orientations, in-plane arrangements, surface terminations, and chemical compositions. The coexistence of strong $\mathrm{Cu}-\mathrm{N}$ ionic bonding and $\mathrm{Ta}-\mathrm{Cu}$ covalent/metallic bonding dictates the stable interfacial structures. Using nudged elastic band method, we calculate the diffusion energy barriers of $\mathrm{Cu}$ to the pre-existing vacancies across $\mathrm{Cu} / \mathrm{Ta}, \mathrm{Cu} / \mathrm{TaN}$ interfaces, and in bulk TaN compounds. As a comparison, $\mathrm{Cu}$ diffusion in $\mathrm{Si}$ is also studied. It was found that $\mathrm{Cu}$ can easily diffuse into $\mathrm{Si}$ either spontaneously or with small energy barriers. On the other hand, although the $\mathrm{Cu} / \mathrm{Ta}$ interfacial diffusion barrier is low, the high vacancy formation energy in Ta renders $\mathrm{Cu}$ diffusion difficult. We find that fcc $\mathrm{TaN}$ is an excellent candidate for diffusion barrier material owing to its extremely high interfacial diffusion energy barrier. The bulk diffusion barrier of $\mathrm{Cu}$ in fcc TaN is also very high.
\end{abstract}

DOI: 10.1103/PhysRevB.79.214104

PACS number(s): 68.35.Fx, 71.20.Be, 31.15.A-

\section{INTRODUCTION}

Copper $(\mathrm{Cu})$ has been widely accepted as an attractive material for interconnects in ultralarge-scale integrated (ULSI) circuits owing to its excellent electrical conductance and higher resistance to electromigration compared to $\mathrm{Al}$ and $\mathrm{Al}$ alloys. However, it is also well known that $\mathrm{Cu}$ atoms diffuse quickly into $\mathrm{Si}$ substrate and $\mathrm{SiO}_{2}$ dielectric, which causes degradation of transistor reliability by forming impurity levels in the band structure, detrimental to the electrical properties of the transistor. ${ }^{1}$ Therefore the integration of an effective diffusion barrier layer between $\mathrm{Cu}$ and $\mathrm{Si}$ substrates to prevent the interdiffusion and reaction becomes crucially important for the ULSI devices. ${ }^{2-4}$

Many materials have been considered as diffusion barriers in $\mathrm{Cu}$ metallization systems, including refractory metals and their nitrides. Among them, tantalum (Ta) and tantalum nitrides (TaN) have received the most attentions owing to their high thermal stability and resistance to form compounds with $\mathrm{Cu}^{3} \mathrm{TaN}$ system including $\mathrm{Ta}, \mathrm{Ta}_{2} \mathrm{~N}$, and $\mathrm{TaN}$ have been selected as the diffusion barrier layers in experimental studies, and thus they are the focus of the present theoretical effort. On the experimental side, research is mostly concentrated on the growth of TaN thin films, the characterization of fabricated structures, and the annealing test of barrier effectiveness. ${ }^{5-14}$ It has been shown that the presence of $\mathrm{N}$ in TaN compounds enhances the resistivity to diffusions ${ }^{6,7}$ and the stacking of TaN compounds on Ta layers ${ }^{11}$ improves the performance of the barrier materials. However the relationship between the barrier efficiency and material structure has not been established. On the theoretical side, research has focused on bulk properties ${ }^{15}$ and electronic structures of TaN compounds, ${ }^{16-18}$ the thermal stability of $\mathrm{Cu} / \mathrm{TaN} / \mathrm{Si}$ stacking structures, ${ }^{19}$ and statistical modeling of atomic diffusions. ${ }^{20}$ However, the crucial issues such as why and how TaN compounds work as diffusion barrier materials are still not well understood. Here we present comprehensive first-principles study to understand the atomistic mechanism of $\mathrm{Cu}$ diffusion into TaN compounds and $\mathrm{Si}$ substrates.

In Sec. II, we present the structural models and computational method for the relevant interfaces. The results are pre- sented and discussed in Sec. III. The bulk properties of Ta and TaN compounds ${ }^{5-7,10-14}$ are presented in Sec. III A while the interfacial properties of $\mathrm{Cu} / \mathrm{TaN}$ system are included in Sec. III B. The interfacial and bulk diffusion barriers are calculated in Sec. III C, followed by a summary in Sec. IV.

\section{CRYSTAL STRUCTURE AND COMPUTATIONAL METHOD}

Four types of TaN crystalline structures are considered as candidates of diffusion barrier materials: bcc Ta, $\delta$-TaN with fcc structure, hexagonal $\epsilon$-TaN, and hexagonal $\mathrm{Ta}_{2} \mathrm{~N}$, shown in Fig. 1. Other relevant materials, such as $\beta$-Ta are not included in the present study because they are not thermodynamically stable. However, these materials may also be used as diffusion barriers under special conditions. ${ }^{21}$ For each of them, $\mathrm{Cu} /$ barrier material interfaces are constructed by depositing $\mathrm{Cu}$ thin layers on top of the barrier substrates. The

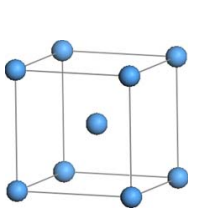

(a)

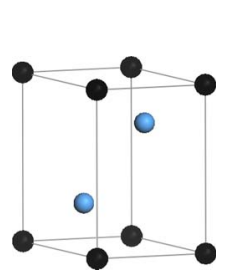

(d)

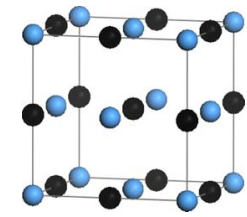

(b)

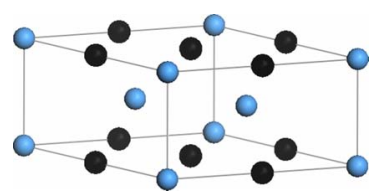

(c)
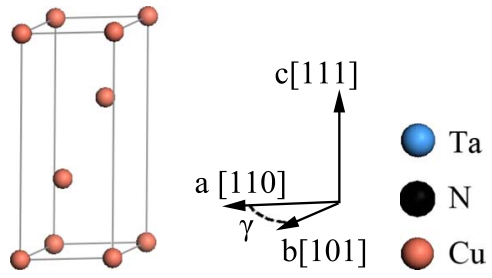

(e)

FIG. 1. (Color online) Crystal structure of Ta, TaN compounds, and $\mathrm{Cu}$ : (a) bcc Ta, (b) fcc TaN, (c) hexagonal TaN, (d) hexagonal $\mathrm{Ta}_{2} \mathrm{~N}$, and (e) fcc $\mathrm{Cu}$, and relevant orientations. $\gamma$ is the angle between two horizontal axis. 
detailed interfacial structures will be discussed in Sec. III B.

Ultrathin $\mathrm{Cu}$ films deposited on bcc Ta (111) have been studied recently. ${ }^{22-26}$ It is generally believed that $\mathrm{Cu}$ grows on Ta (110) substrate in a layer-by-layer mode for a small number of monolayers and then switches to a threedimensional (3D) growth model. The relation between the number of stable $\mathrm{Cu}$ monolayers and the instability point is unclear. It is clear however from both experimental and theoretical results that $\mathrm{Cu}$ forms stable pseudomorphic films on top of Ta, i.e., films which have the same structure as the Ta substrate. ${ }^{21,23}$ Since 3D simulations of $\mathrm{Cu}$ islands or droplets on Ta based on first-principles are not feasible computationally, we focus on the pseudomorphic thin-film mode in this study. This is a reasonable approximation because the interfacial diffusions are highly localized, taking place near the interface. Monolayer of $\mathrm{Cu}$ is not a good choice for the diffusion barrier calculations because it introduces artificial $\mathrm{Cu}$ surface which is not present in reality. On the other hand, a pseudomorphic thin film with too many $\mathrm{Cu}$ monolayers is not realistic either because only a very small number of $\mathrm{Cu}$ layers are observed to be stable on Ta. Therefore, we decide to include three $\mathrm{Cu}$ (111) layers on top of Ta (110) substrate in the interfacial diffusion calculations. fcc TaN was reported to be a metastable structure ${ }^{16,27}$ but it is believed to be the most effective diffusion barrier material among all TaN compounds. $^{27}$ The surface orientation of fcc TaN differs depending on the methods of fabrication; ${ }^{28}$ it is reported however that the densely packed (111) orientation is considered the most effective orientation for barrier materials. The $\mathrm{Cu} /$ fcc-TaN interface structures remain unclear experimentally except that $\mathrm{Cu}(111)$ has been reported as preferred orientation on fcc-TaN substrate. ${ }^{8-14,27,28}$ Therefore, we will study $\mathrm{Cu}$ (111)/fcc-TaN (111) interfaces by including three $\mathrm{Cu}$ (111) layers on top of TaN (111) substrate in the firstprinciples calculations. ${ }^{29-32}$

All calculations in this paper are carried out based on first-principles density-functional theory (DFT) with planewave basis and the projector augmented-wave method as implemented in the Vienna $A b$ initio Simulation Package. Nonrelativistic ultrasoft pseudopotential ${ }^{33}$ and the generalized gradient approximation ${ }^{34}$ are used in the calculations. The energy cutoff of Ta, TaN compounds, $\mathrm{Cu}$, and $\mathrm{Si}$ is 175 , 348, 234, and $151 \mathrm{eV}$, respectively. The Fermi-Dirac smearing is used for all cases except for bulk calculations where the tetrahedron method is employed instead; the smearing energy is $0.2 \mathrm{eV}$ throughout. For bulk calculations of bcc Ta, fcc $\mathrm{TaN}$ and $\mathrm{Si}$, and hexagonal $\mathrm{TaN}$ and hexagonal $\mathrm{Ta}_{2} \mathrm{~N}$, the Monkhorst-Pack meshes of $15 \times 15 \times 15,11 \times 11 \times 15$, and $15 \times 15 \times 11$ are used, respectively.

The adhesive strength of the $\mathrm{Cu} /$ substrate interfaces are evaluated with the interfacial cohesive energy $\gamma_{c}$ defined as the work per unit area that is required to separate the $\mathrm{Cu} /$ substrate interfaces into isolated $\mathrm{Cu}$ layers and the substrate

$$
\gamma_{c}=\frac{E_{\mathrm{int}}-E_{\infty}}{A} .
$$

Here $E_{\text {int }}$ is the total energy of the $\mathrm{Cu}$ /substrate combined system, and $E_{\infty}$ is the total energy when $\mathrm{Cu}$ and the substrate are separated to infinity. $A$ is the area of the interface in the computational unit cell.

The $k$-point mesh of $11 \times 11 \times 1$ is used for the interfacial calculations of $\mathrm{Cu} / \mathrm{fcc} \mathrm{Ta}, \mathrm{Cu} /$ hexagonal $\mathrm{Ta}_{2} \mathrm{~N}$, and $\mathrm{Cu} / \mathrm{bcc}$ Ta; $8 \times 8 \times 1$ mesh is used for $\mathrm{Cu} /$ hexagonal TaN. The strain energy of $\mathrm{Cu}$ on the substrates is calculated separately by using a laterally strained unit cell.

The cohesive energy of the elemental bulk system is evaluated by subtracting the atomic energy from the total energy of the crystal. The atomic energy is calculated by using a large unit cell of $20 \AA$ with spin-polarization corrections. The cohesive energy of TaN compounds is determined by subtracting the chemical potential of Ta and $\mathrm{N}$ atoms from the total energy. The chemical potential of $\mathrm{Ta}$ and $\mathrm{N}$ is determined with respect to bcc-Ta and free $\mathrm{N}_{2}$ molecules, respectively.

The vacancy formation energy $\left(E_{f}\right)$ is calculated for various materials. For the elemental materials, $E_{f}$ is defined as

$$
E_{f}=E_{n-1}-\frac{(n-1)}{n} E_{n},
$$

where $E_{n}$ is the energy of the perfect bulk with $n$ atoms in the unit cell, and $E_{n-1}$ is the energy of this system with a vacancy. For binary compounds, the formation energy of Ta and $\mathrm{N}$ vacancies, $E_{f}^{\mathrm{Ta}}$ and $E_{f}^{\mathrm{N}}$, can be calculated by

$$
\left\{\begin{array}{l}
E_{f}^{\mathrm{Ta}}=E_{n-1}^{\mathrm{Ta}-\mathrm{vac}}-E_{n}+\mu^{\mathrm{Ta}}, \\
E_{f}^{\mathrm{N}}=E_{n-1}^{\mathrm{N}-\mathrm{vac}}-E_{n}+\mu^{\mathrm{N}},
\end{array}\right.
$$

where $\mu^{\mathrm{Ta}}$ and $\mu^{\mathrm{N}}$ are chemical potentials of Ta and $\mathrm{N}$ in the TaN compound, $E_{n}$ is the energy of perfect bulk system with $n$ atoms, and $E_{n-1}^{\mathrm{Ta}-\mathrm{ac}}$ and $E_{n-1}^{\mathrm{N}-\mathrm{vac}}$ are the energy of the bulk system with a Ta or $\mathrm{N}$ vacancy, respectively. Unfortunately, the chemical potential of $\mathrm{Ta}$ or $\mathrm{N}$ atom is not well defined depending on the chemical environment of the atom. However, the chemical potentials should satisfy the following equation:

$$
x \mu^{\mathrm{Ta}}+y \mu^{\mathrm{N}}=E_{n} .
$$

$x$ and $y$ are the number of Ta and $\mathrm{N}$ atoms in the unit cell of the perfect bulk lattice with $x+y=n$. With this equation, we can estimate the range of the chemical potentials based on the fact that the chemical potential of each species should be lower than that of their corresponding elemental phaseotherwise the elemental phase is preferred over the binary compound. Taking the chemical potential of Ta to that of bcc Ta and the chemical potential of $\mathrm{N}$ to that of free $\mathrm{N}_{2}$ molecule, we can estimate the range of $E_{f}$.

The interfacial diffusion barriers are calculated by displacing a $\mathrm{Cu}$ atom to a pre-existing vacancy across the interface. The minimum-energy path and the corresponding energy barrier are determined by using the nudged elastic band (NEB) method. The bulk diffusion barriers are also calculated similarly by moving a $\mathrm{Cu}$ atom to a pre-existing bulk vacancy along different directions.

In NEB calculations, the spring constant is $5 \mathrm{eV} / \AA^{2}$ and the images are optimized using the quasi-Newton method. $4 \times 8 \times 1$ and $6 \times 6 \times 1 k$-point meshes are used in the calculations of $\mathrm{Cu} / \mathrm{TaN}$ and $\mathrm{Cu} / \mathrm{Si}$ interfaces, respectively. The 
TABLE I. Lattice constant $(\AA)$ and cohesive energy $E_{c}(\mathrm{eV} /$ atom) of various materials.

\begin{tabular}{|c|c|c|c|}
\hline Structure & Lattice constant & $E_{c}$ (this work) & $E_{c}$ (reference) \\
\hline $\mathrm{fcc} \mathrm{Cu}$ & 3.644 & -3.53 & $-3.49^{\mathrm{a}}$ \\
\hline Diamond Si & 5.458 & -4.64 & $-4.63^{a}$ \\
\hline bcc Ta & 3.298 & -8.30 & $-8.10^{\mathrm{a}}$ \\
\hline fcc TaN & 4.406 & -1.00 & $-1.305^{\mathrm{b}}$ \\
\hline Hex TaN & $a=5.238 \quad c=2.913$ & -0.82 & $-1.117^{b}$ \\
\hline $\operatorname{Hex} \mathrm{Ta}_{2} \mathrm{~N}$ & $a=3.099 \quad c=4.852$ & -0.88 & $-1.086^{\mathrm{b}}$ \\
\hline
\end{tabular}

${ }^{\mathrm{a} F r o m ~ R e f . ~} 35$.

${ }^{b}$ From Ref. 16.

bulk diffusion calculations are carried out with $4 \times 4 \times 4 k$ mesh except for diffusion along [111] direction in fcc TaN in which a $4 \times 6 \times 6$ mesh is used.

\section{RESULT AND DISCUSSION}

Four factors are taken into consideration for constructing $\mathrm{Cu}$ /substrate interface models: (a) crystalline structure of the substrates, (b) relative orientation of the substrates, (c) terminating layer of the substrates, and (d) in-plane arrangement at the interface. The optimal interfacial structure is determined by exploring the combination of the four factors with the lowest interfacial cohesive energy. Having obtained the optimal interfacial structures, we can carry out relevant diffusion barrier calculations.

\section{A. Bulk properties}

In this paper, we focus on bcc Ta and three types of TaN compounds, including fcc $\delta$-TaN, hexagonal (hex-) $\epsilon$-TaN, and hexagonal (hex-) $\mathrm{Ta}_{2} \mathrm{~N}$; they have one Ta atom, one $\mathrm{Ta}$ and one $\mathrm{N}$ atom, three Ta and three $\mathrm{N}$ atoms, and two Ta and one $\mathrm{N}$ atoms per unit cell, respectively, shown in Fig. 1. The results of their bulk properties are summarized in Table I, which also includes the bulk properties of $\mathrm{Si}$ and $\mathrm{Cu}$.

The bulk properties of $\mathrm{Cu}, \mathrm{Si}$, and Ta agree well with the experimental data. ${ }^{35}$ The difference in cohesive energy of TaN compounds compared to that of Stampfl and Freeman ${ }^{16}$ may be due to the difference in chemical potential of $\mathrm{N}$ between our result and that of Stampfl (there is no report of $\mathrm{N}$ chemical potential in Ref. 16). If the chemical potential of $\mathrm{N}$ in our calculation is shifted by $0.6 \mathrm{eV}$, the cohesive energy of fcc $\mathrm{TaN}$, hexagonal $\mathrm{TaN}$, and hexagonal $\mathrm{Ta}_{2} \mathrm{~N}$ becomes $-1.30,-1.13$, and $-1.08 \mathrm{eV}$, respectively, which agree well with Stampfl and Freeman. ${ }^{16}$

\section{B. Interfacial structures}

In this work, we take the experimentally observed orientations of $\mathrm{Cu} /$ barrier interface: $\mathrm{Cu}$ (111) layers on top of (111) surface of fcc TaN, ${ }^{8-14}$ (110) surface of bcc Ta, ${ }^{9,10,14}$ (001) surface of hexagonal $\mathrm{Ta}_{2} \mathrm{~N},{ }^{5}$ and (001) surface of hexagonal TaN. The interfacial cohesive energy is calculated as a function of terminating surface and in-plane arrangement for each of the substrates. First, we determine the lattice
TABLE II. Lattice parameters and strain energy per atom $\Delta E$ $(\mathrm{eV})$ of the substrates. The strain is due to the mismatch between $\mathrm{Cu}$ (111) surface and the surface of the various substrates listed in the table.

\begin{tabular}{lcccc}
\hline \hline Substrate & $\begin{array}{c}a \\
(\AA)\end{array}$ & $\begin{array}{c}c \\
(\AA)\end{array}$ & $\begin{array}{c}\gamma \\
\left({ }^{\circ}\right)\end{array}$ & $\Delta E$ \\
\hline Unstrained & 2.58 & 6.31 & 60.0 & \\
fcc TaN (111) & 3.11 & 5.17 & 60.0 & 0.33 \\
bcc Ta (110) & 2.86 & 5.54 & 70.5 & 0.26 \\
Hex TaN (001) & 2.62 & 6.23 & 60.0 & 0.01 \\
Hex Ta 2 N (001) & 3.10 & 5.21 & 60.0 & 0.33 \\
Si (100) & 3.86 & 3.31 & 90.0 & 0.04 \\
Si (111) & 2.23 & 6.99 & 60.0 & 0.68 \\
\hline \hline
\end{tabular}

parameters and cohesive energy of strained $\mathrm{Cu}$ lattice and the results are summarized in Table II.

The interfacial structure is constructed by placing a threelayer $\mathrm{Cu}$ slab on top of a six-layer of substrate slab, with a separation of $2 \AA$ in between. A $30 \AA$ vacuum is included to separate the periodic images along the surface-normal direction. To calculate $E_{\text {int }}$, the last two layers of the substrate are fixed while the rest of the system is allowed to relax until the energy difference between two consecutive configurations is less than $10^{-4} \mathrm{eV} . E_{\infty}$ is obtained by separating the $\mathrm{Cu}$ slab and the substrate of $15 \AA$, and then relaxing the structure. Convergence test including 12 layers of substrate yields an interfacial cohesive energy that is only $0.05 \mathrm{~J} / \mathrm{m}^{2}$ from the much smaller model. The good agreement between the two different choices of supercell suggests that interfacial cohesion is short ranged and primarily localized at the interface. The schematic diagrams of interfacial structure and the calculated cohesive energy are shown in Fig. 2.

\section{Cu/fcc-TaN interface}

The pseudomorphic interface is constructed by straining the two-dimensional (2D) unit cell of $\mathrm{Cu}$ (111) surface to match the 2D unit cell of fcc-TaN (111) surface, with $\mathrm{Cu}$ [110] direction parallel to fcc-TaN [110] direction. Five inplane arrangements are examined, in which $\mathrm{Cu}$ atoms sit on various symmetric sites above the substrate: (1) top sites, (2) fcc sites, (3) hcp sites, (4) half way between the top and fcc sites, and (5) bridge sites, summarized in Fig. 2(a). For each of the in-plane arrangement, two types of surface termination of TaN are considered: Ta and $\mathrm{N}$ termination. It is found that $\mathrm{Cu}$ atoms prefer to sit at the threefold fcc and hcp sites above Ta atoms and on top of $\mathrm{N}$ atoms (Fig. 2). The interfacial bond length of $\mathrm{Ta}-\mathrm{Cu}$ and $\mathrm{TaN}$ is 2.72 and $1.93 \AA$, respectively. To understand the nature of interfacial cohesion, we plot the bonding charge density $\rho_{b}(\mathbf{r})$, defined as

$$
\rho_{b}(\mathbf{r})=\rho_{\text {solid }}(\mathbf{r})-\sum_{i} \rho_{\text {at }}\left(\mathbf{r}-\mathbf{R}_{i}\right),
$$

in Fig. 3. Here $\rho_{\text {solid }}$ is the solid charge density of the system and $\rho_{\mathrm{at}}(\mathbf{r})$ is the atomic charge density centered at atom $i$, whose position is given by $\mathbf{R}_{i}$. The density of states (DOS) of interfacial atoms is shown in Fig. 3. For Ta-terminating sur- 
(1)

(a)

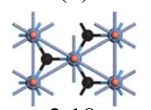

(b)

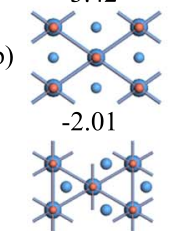

(c)

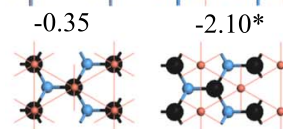

$-1.44 *$

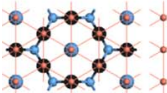

(d)

$-2.34 *$

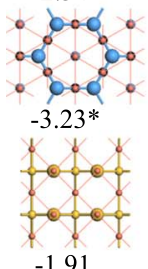

(f)

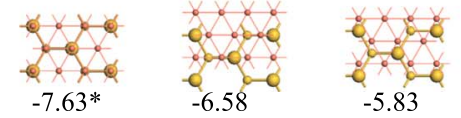

FIG. 2. (Color online) Schematic of interfacial structure and the corresponding interfacial cohesive energy, from top to bottom: (a) $\mathrm{Cu}(111) / \mathrm{fcc} \mathrm{TaN}$ (111) with Ta termination, $\mathrm{Cu}$ (111)/fcc TaN (111) with $\mathrm{N}$ termination, (b) $\mathrm{Cu}$ (111)/bcc Ta (110), (c) $\mathrm{Cu}$ (111)/hex $\mathrm{Ta}_{2} \mathrm{~N}(001)$ with Ta termination, $\mathrm{Cu}(111) /$ hex $\mathrm{Ta}_{2} \mathrm{~N}(001)$ with $\mathrm{N}$ termination, (d) $\mathrm{Cu}$ (111)/hex TaN (001) with TaN termination, $\mathrm{Cu}$ (111)/hex-TaN (001) with Ta termination, (e)Cu (100)/Si (100), and (f) $\mathrm{Cu}(111) / \mathrm{Si}$ (111). Only one layer of $\mathrm{Cu}$ atoms and two layers of the substrates are shown. The size of the spheres is scaled so that the larger the spheres, the closer the atoms to the interface (the largest spheres represent the atoms at the interface). The $\mathrm{Cu}$ spheres are scaled down to allow the substrate visible. The orientation of each interface is also shown in the figure. The interfacial cohesive energy $\left(\mathrm{J} / \mathrm{m}^{2}\right)$ is labeled under each atomic configuration, with the lowest energy configuration for each substrate marked by an asterisk.

face, there is strong metallic bonding between a $\mathrm{Cu}$ atom and three neighboring Ta atoms. The metallic bonding across the interface, consisting of $\mathrm{Cu} 4 s$ and Ta $6 s$ states, is much stronger than the metallic bonding inside the $\mathrm{Cu}$ lattice. For $\mathrm{N}$-terminating surface, strong ionic bonding is developed between $\mathrm{Cu}$ and $\mathrm{N}$ across the interface, with charge transferred from $\mathrm{Cu}$ to $\mathrm{N}$. The ionic bonding between $\mathrm{Cu}$ and $\mathrm{N}$ is stronger than that between $\mathrm{Ta}$ and $\mathrm{N}$. The bonding naturemetallic vs ionic across the interface-dictates the stable interfacial structures.
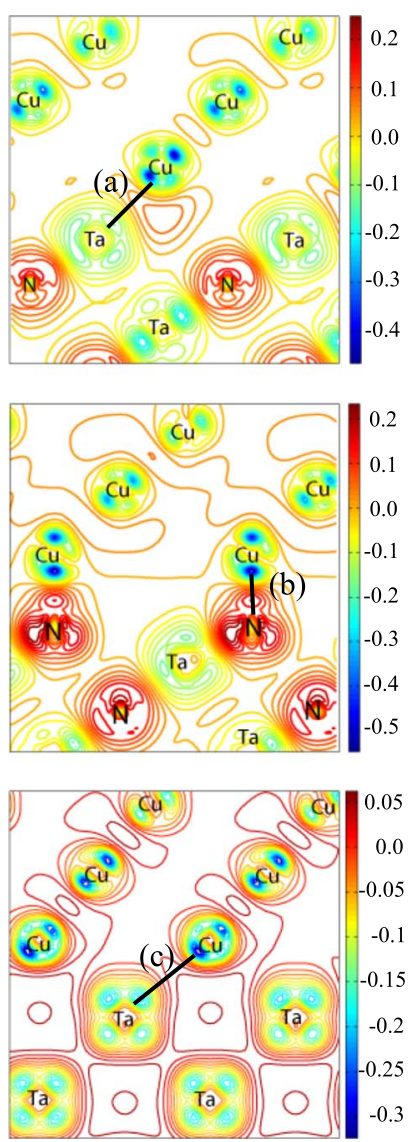

(a)

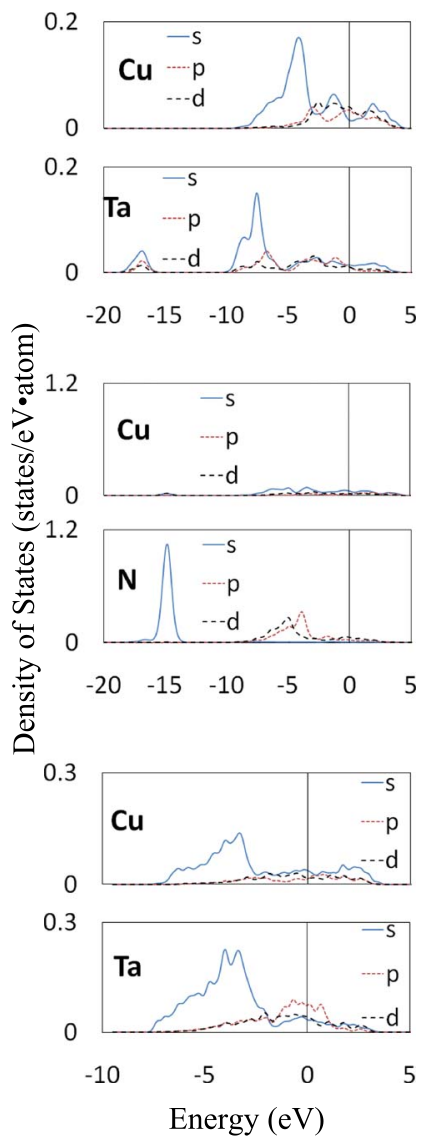

(b)

FIG. 3. (Color online) Left, bonding charge density (in $\AA^{-3}$ ) near the interface: from top to bottom, $\mathrm{Cu} / \mathrm{fcc} \mathrm{TaN}$ with Ta termination, $\mathrm{Cu} / \mathrm{fcc}$ TaN with $\mathrm{N}$ termination, and $\mathrm{Cu} / \mathrm{bcc}$ Ta. The atoms that are connected by a solid line are of interest, and their local DOS is shown on the right. The angular moment projected DOS is also shown.

\section{Cu/bcc-Ta interface}

The 2D unit cell of $\mathrm{Cu}(111)$ surface is strained and placed on top of bcc-Ta (110) surface with $\mathrm{Cu}[11 \overline{2}]$ direction parallel to $\mathrm{Ta}[1 \overline{1} 0]$ direction. ${ }^{23}$ Because $[001]$ and $[1 \overline{1} 1]$ directions are no longer equivalent, one more type of bridge site has to be considered for the (110) surface of bcc Ta. There are five in-plane arrangements to be explored: (1) top sites, (2) threefold sites where $\mathrm{Cu}$ atoms sit at one third of the long diagonal of Ta (110) unit cell, (3) half way between (1) and (2), (4) bridge sites along [001] direction, and (5) bridge sites along [1시] direction [Fig. 2(b)]. We find that $\mathrm{Cu}$ atoms prefer the bridge sites above Ta atoms along [001] direction and the interfacial Ta-Cu bond length is $2.66 \AA$. This result agrees with that of Hashibon et al. ${ }^{23}$ The bonding charge density and density of states of the interface are presented in Fig. 3: the interfacial cohesion is primarily due to a mixture of covalent/metallic bonding between $\mathrm{Cu} 4 s$ and $\mathrm{Ta} 6 s$ states. These states are delocalized around the interface.

\section{Cu/hex- $-\mathrm{Ta}_{2} \mathrm{~N}$ interface}

The $\mathrm{Cu}$ (111) 2D unit cell is strained to fit the hexagonal $\mathrm{Ta}_{2} \mathrm{~N}(001) 2 \mathrm{D}$ unit cell, with $\mathrm{Cu}[1 \overline{1} 0]$ direction parallel to 
$\mathrm{Ta}_{2} \mathrm{~N}$ [100] direction. $\mathrm{Ta}_{2} \mathrm{~N}$ (001) surface is similar to fcc$\mathrm{TaN}$ (111) surface-both of them have hexagonal unit cells with either Ta or $\mathrm{N}$ termination. The in-plane arrangements of the two interfaces are also similar as shown in Fig. 2(c). It is found that $\mathrm{Cu}$ atoms prefer the top sites on $\mathrm{N}$ atoms and fcc sites above $\mathrm{Ta}$. The $\mathrm{Cu}-\mathrm{Ta}$ and $\mathrm{Cu}-\mathrm{N}$ bond lengths are 2.65 and $1.94 \AA$, respectively.

\section{Cu/hex-TaN interface}

The hexagonal TaN has a much larger unit cell so that 2 $\times 2 \mathrm{Cu}(111) 2 \mathrm{D}$ unit cell can be placed on top of each $\mathrm{TaN}$ (001) 2D unit cell, with $\mathrm{Cu}[1 \overline{10}]$ direction parallel to TaN [100] direction. There are two types of termination of TaN (001) surface: TaN and Ta. Three in-plane arrangements are considered, in which $\mathrm{Cu}$ atoms sit on, referring to the TaN surface: (1) top sites, (2) threefold (including both fcc and hcp) sites, and (3) bridge sites, shown in Fig. 2(d). We find that for TaN termination which has a 1:3 Ta to $\mathrm{N}$ ratio, $\mathrm{Cu}$ atoms prefer to sit on top of both Ta and $\mathrm{N}$ atoms (see Fig. 2). The interfacial $\mathrm{Cu}$ layer develops a zigzag structure due to the different bond length of $\mathrm{Cu}-\mathrm{Ta}(2.35 \AA)$ and $\mathrm{Cu}-\mathrm{N}$ $(1.98 \AA)$. For Ta termination, 3/4 of the interfacial $\mathrm{Cu}$ atoms sit on the bridge sites and $1 / 4$ of the $\mathrm{Cu}$ atoms sit on top of exposed $\mathrm{Ta}$ atoms at the subsurface. The $\mathrm{Cu}-\mathrm{Ta}$ bond length is 2.73 and $2.65 \AA$, respectively.

Based on above discussions, the in-plane arrangements of the $\mathrm{Cu} / \mathrm{TaN}$ interfacial structures could be summarized as follows: for $\mathrm{N}$-terminated surface, $\mathrm{Cu}$ atoms prefer to sit directly on top of $\mathrm{N}$ atoms to form strong ionic bonds; for Ta-terminated surface, $\mathrm{Cu}$ atoms take either the fcc or bridge sites to form a mixture of covalent and metallic bonds. In this case, the interfacial $\mathrm{Cu}$ layer maintains the lattice structure of the substrate - as an extension of the substrate. For the mixed surface termination, the in-plane arrangements follow the same rules with respect to the majority species of the surface atoms.

\section{Cu/Si interface}

It is known that $\mathrm{Cu}$ reacts with $\mathrm{Si}$ to form $\mathrm{Cu}_{3} \mathrm{Si}$ intermetallic compound ${ }^{2,36}$ at the interface, rendering a complicated $\mathrm{Cu} / \mathrm{Si}$ interfacial structure unattainable by first-principles calculations. Here, we focus on unreconstructed $\mathrm{Cu} / \mathrm{Si}$ interfaces instead with two orientations: $\mathrm{Cu}$ (111)/Si (111) (Refs. 37 and 38) and $\mathrm{Cu}$ (100)/Si (100) (Ref. 39); both of them have been examined experimentally. Our modest goal here is to determine the simplistic "perfect" interface for which $\mathrm{Cu}$ diffusion barriers can be extracted and compared to $\mathrm{Cu} / \mathrm{TaN}$ system. Similar to the procedures outlined for TaN compounds, various in-plane arrangements are considered. The results show that the (111) interface has a much lower cohesive energy than the (100) interface, thus energetically more stable. $\mathrm{Cu}$ atoms prefer the bridge sites on $\mathrm{Si}$ (100) surface and a mixture of top, fcc, and hep sites on $\mathrm{Si}$ (111) surface [Figs. 2(e) and 2(f)].

Because fcc-TaN and bcc-Ta substrates provide stronger adhesive power to $\mathrm{Cu}$ than other materials, they are potentially better candidates for diffusion barrier materials. In addition, much more experimental work on the two materials
TABLE III. Vacancy formation energy $E_{f}(\mathrm{eV})$ in bulk $\mathrm{Cu}$, bulk diamond-Si, bcc-Ta, and fcc-TaN. For fcc-TaN, the range of $E_{f}$ is presented, corresponding to $\mathrm{N}$-rich and Ta-rich conditions. The number of atoms in the computational unit cell is also listed.

\begin{tabular}{|c|c|c|c|c|}
\hline Material & Atoms per cell & & $E_{f}$ & $E_{f}$ in Refs. \\
\hline $\mathrm{fcc} \mathrm{Cu}$ & 108 & & 1.04 & $1.28^{\mathrm{a}}$ \\
\hline Diamond Si & 64 & & 3.66 & $3.53^{\mathrm{b}}$ \\
\hline bcc Ta & 128 & & 2.96 & $2.95^{\mathrm{c}}$ \\
\hline $\mathrm{N}$ vacancy in fcc TaN & 64 & 0.84 & -1.15 & \\
\hline Ta vacancy in fcc TaN & 64 & -3.09 & -1.10 & \\
\hline
\end{tabular}

aFrom Refs. 40 and 41.

${ }^{b}$ From Ref. 42.

${ }^{\mathrm{c}}$ From Refs. 41 and 43.

have been reported, we therefore focus on fcc TaN and bcc Ta for diffusion barrier calculations in the next section.

\section{C. $\mathrm{Cu}$ diffusion barriers}

\section{Vacancy formation energy}

Due to the large size of $\mathrm{Cu}$ atoms, interfacial and bulk diffusions of $\mathrm{Cu}$ will normally involve vacancies-diffusion via interstitials is energetically highly unfavorable. We start by calculating vacancy formation energy for various materials and the results are summarized in Table III. It is found that the vacancy formation energy of $\mathrm{Si}$ and $\mathrm{Ta}$ is very large-much larger than that in $\mathrm{Cu}$, suggesting the necessity of pre-existing vacancies for diffusion in $\mathrm{Si}$ and $\mathrm{Ta}$. As alluded before, the vacancy formation energy of TaN compounds depends on the chemical potential of each species. We estimate the range of the vacancy formation energy of the compounds by imposing the constraint ${ }^{44}$ that the chemical potential of $\mathrm{Ta}$ and $\mathrm{N}$ in a TaN compound should not be greater than that in bcc-Ta and free $\mathrm{N}_{2}$ molecules. The extreme case of equality of the chemical potential in the elemental and the binary phase for one species indicates the rich condition of this element. Interestingly, the Ta-vacancy formation energy of in fcc $\mathrm{TaN}$ is always negative, ranging from -3.09 ( $\mathrm{N}$ rich) to $-1.10 \mathrm{eV}$ (Ta rich), and $\mathrm{N}$-vacancy formation energy in fcc TaN ranges from 0.84 ( $\mathrm{N}$ rich) to $-1.15 \mathrm{eV}$ (Ta rich). The results suggest that vacancies are actually favored in fcc TaN, especially with Ta vacancy. The results agree with the finding of Stampfl and Freeman ${ }^{16}$ that $\mathrm{Ta}_{4} \mathrm{~N}_{5}$, which is an fcc structure with ordered Ta vacancies, is more stable than fcc TaN.

In the NEB calculations, the initial state is the interfacial structures determined in the previous section, with a vacancy introduced in the substrate across the interface. The final state involves the same interfacial structure but with the nearest-neighbor $\mathrm{Cu}$ atom displaced to the vacancy in the substrate, leaving behind a vacancy in $\mathrm{Cu}$. Before proceeding to NEB calculations, both the initial and final states are relaxed by the conjugate-gradient method until the energy is converged to $10^{-3} \mathrm{eV}$. The $\mathrm{Cu}$ top layer and the last two layers of the substrate are held fixed in the relaxation and NEB calculations. The bulk diffusion barriers are also calcu- 


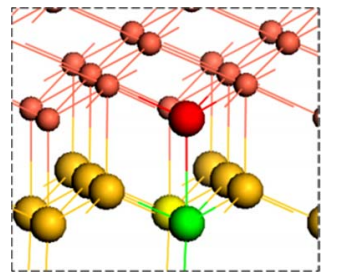

(a)

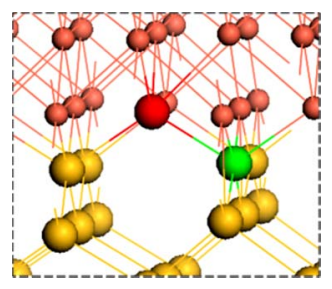

(b)
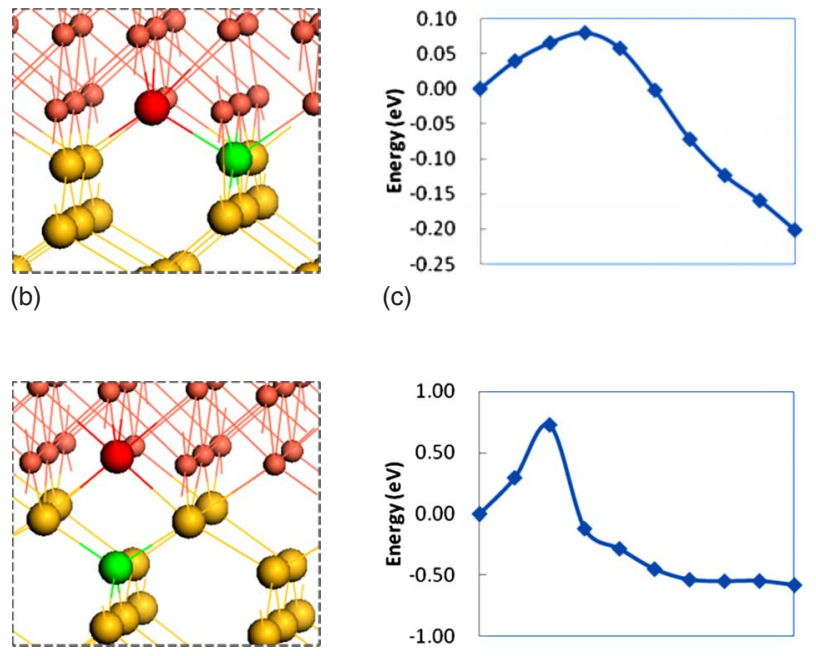

(d) (c)
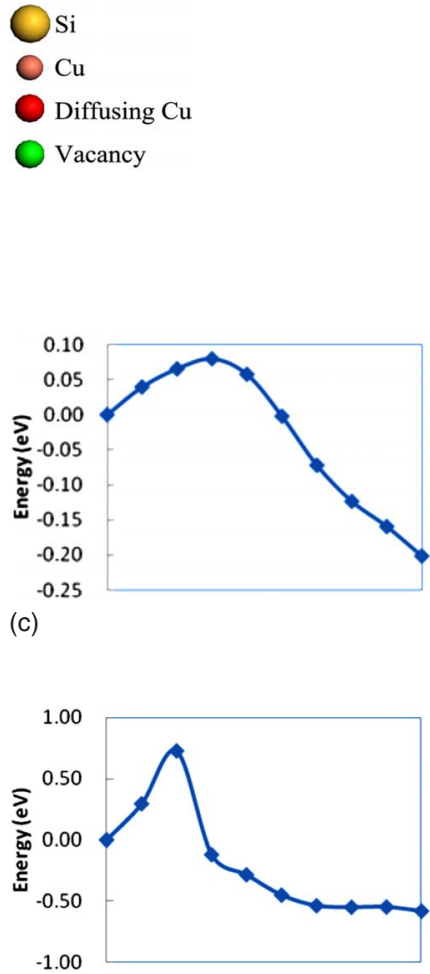

(e)

FIG. 4. (Color online) $\mathrm{Cu} / \mathrm{Si}$ interfacial diffusions: (a) $\mathrm{Cu}$ (111)/Si (111) interfacial structure with a diffusing $\mathrm{Cu}$ atom and a $\mathrm{Si}$ vacancy at the interface. The $\mathrm{Cu}$ atom diffuses into the $\mathrm{Si}$ vacancy spontaneously during the relaxation. (b) $\mathrm{Cu}(100) / \mathrm{Si}$ (100) interfacial structure with a diffusing $\mathrm{Cu}$ atom and a $\mathrm{Si}$ vacancy at the interface. The $\mathrm{Cu}$ atom diffuses into the vacancy with $0.07 \mathrm{eV}$ energy barrier. The energy profile for the diffusion is shown in (c). (d) $\mathrm{Cu}(100) / \mathrm{Si}(100)$ interfacial structure with a diffusing $\mathrm{Cu}$ atom and a $\mathrm{Si}$ vacancy at one layer below the interface. The $\mathrm{Cu}$ atom diffuses into the vacancy with $0.73 \mathrm{eV}$ energy barrier. The energy profile of the diffusion is shown in (e).

lated for Ta and TaN compounds in the similar manner: two or more adjacent vacancies are introduced in the bulk, and a $\mathrm{Cu}$ atom is placed at one of the vacancies as the initial state and at the adjacent vacancy as the final state.

\section{Cu/Si interfacial diffusion}

For $\mathrm{Cu}(111) / \mathrm{Si}$ (111) interface, periodic boundary condi-

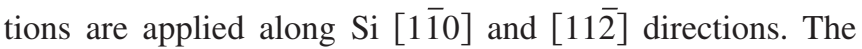
supercell contains three $\mathrm{Cu}$ and eight $\mathrm{Si}$ layers in which there are $31 \mathrm{Si}$ and $36 \mathrm{Cu}$ atoms; a vacuum slab of $15 \AA$ is included. A Si vacancy is introduced right below a $\mathrm{Cu}$ atom [see Fig. 4(a)]. We find that, after the relaxation of the initial state, the $\mathrm{Cu}$ atom moves to the $\mathrm{Si}$ vacancy spontaneously with no energy barrier for diffusion. This result is consistent with the fact that $\mathrm{Cu}$ and $\mathrm{Si}$ react easily at the interface. ${ }^{2}$

For $\mathrm{Cu}(100) / \mathrm{Si}(100)$ interface, the interface plane has a $2 \times 2$ unit cell along $\mathrm{Si}[0 \overline{1} 1]$ and [011] directions, for which the periodic boundary conditions are applied. The supercell consists of three $\mathrm{Cu}$ and eight $\mathrm{Si}$ layers, with $31 \mathrm{Si}$ and $24 \mathrm{Cu}$ atoms. Two types of Si vacancy are considered: a fourfold

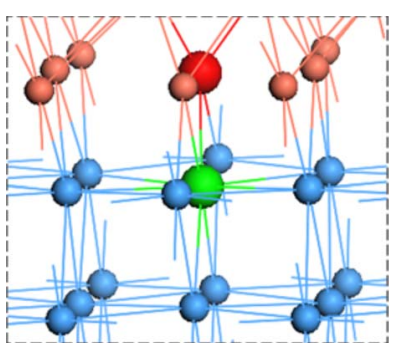

(a)

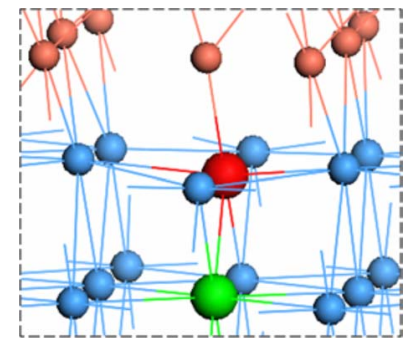

(c)

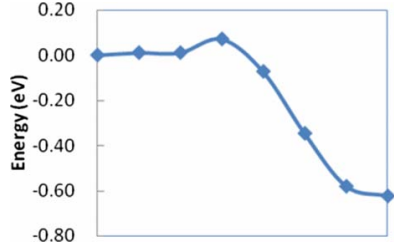

(b)

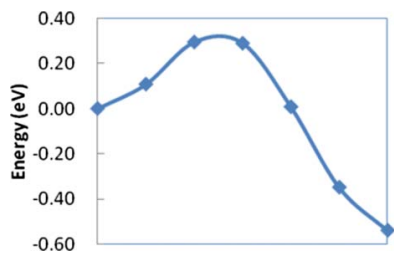

(d)
Ta $\mathrm{O}$ Cu Diffusing $\mathrm{Cu} O$ Vacancy

FIG. 5. (Color online) $\mathrm{Cu} / \mathrm{bcc}-\mathrm{Ta}$ interfacial diffusions: (a) interfacial structure with a diffusing $\mathrm{Cu}$ atom and a Ta vacancy at the interface. The $\mathrm{Cu}$ atom diffuses into the vacancy with $0.07 \mathrm{eV}$ energy barrier. The energy profile for the diffusion is shown in (b). (c) Interfacial structure with a diffusing $\mathrm{Cu}$ atom and a Ta vacancy at one layer below the interface. The $\mathrm{Cu}$ atom diffuses into the vacancy with $0.29 \mathrm{eV}$ energy barrier. The energy profile of the diffusion is shown in (d).

site below $\mathrm{Cu}$ atoms and a site directly below a $\mathrm{Cu}$ atom in the second Si layer [see Fig. 4(b)]. It is found that there is a small energy barrier $(0.07 \mathrm{eV})$ for the $\mathrm{Cu}$ atom diffusing into the first vacancy, and $0.73 \mathrm{eV}$ barrier into the second vacancy.

\section{Diffusion barriers in bcc $T a$}

The $\mathrm{Cu}(111) / \mathrm{Ta}(110)$ interface has a $2 \times 2$ unit cell in Ta [110] and [001] directions, of which the periodic boundary conditions are applied. The supercell includes three $\mathrm{Cu}$ and four Ta layers with $31 \mathrm{Ta}$ and $24 \mathrm{Cu}$ atoms.

First, in the initial state, a vacancy is introduced at the top Ta layer; in the final state, a $\mathrm{Cu}$ atom is moved to the $\mathrm{Ta}$ vacancy. A small energy barrier of $0.07 \mathrm{eV}$ is found for this diffusion process, accompanied by an energy drop of $0.63 \mathrm{eV}$ in the final state, shown in Fig. 5(a). Based on the final state, another vacancy is introduced at the second Ta layer and the $\mathrm{Cu}$ atom is displaced from the first vacancy to the second one. It is found that there is a barrier of $0.29 \mathrm{eV}$ with an energy drop of $0.54 \mathrm{eV}$, shown in Fig. 5(b).

The above results indicate that, as $\mathrm{Cu}$ atom diffuses further into $\mathrm{Ta}$, the energy barrier increases and the final state becomes less favorable. Therefore it would be interesting to examine the "asymptotic" interfacial diffusion behavior: $\mathrm{Cu}$ diffusion in bulk Ta. The diffusion energy barriers are evaluated by moving a $\mathrm{Cu}$ atom between two adjacent vacancies along different directions in bulk Ta. The cubic supercell consisting of $54 \mathrm{Ta}$ atoms is used in the calculation. The 


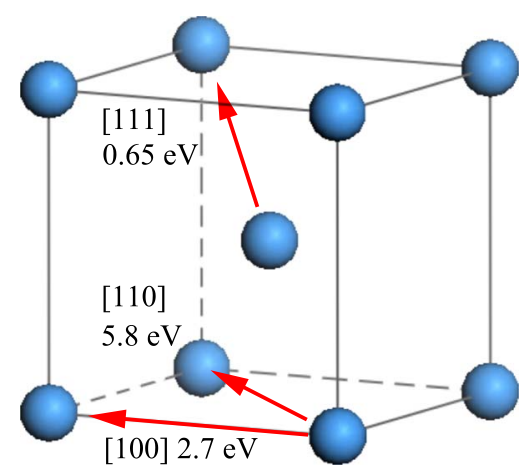

FIG. 6. (Color online) Bulk diffusion barriers of $\mathrm{Cu}$ in bec Ta. The energy barriers along different directions are shown. The arrow points from the diffusing $\mathrm{Cu}$ atom to the vacancy for each direction of interest.

results of bulk diffusion barrier are shown in Fig. 6. The lowest bulk diffusion barrier is $0.65 \mathrm{eV}$ and is along [111] direction. Therefore, we conclude that $\mathrm{Cu}$ diffusion in $\mathrm{Ta}$ becomes increasingly more difficult going from interface to bulk. Furthermore, owing to the large vacancy formation energy, the probability of existing vacancies in Ta is low, which limits the rate of $\mathrm{Cu}$ diffusion in Ta. In other words, bcc Ta could be a good candidate for diffusion barrier material.

\section{Diffusion in fcc TaN}

To model interfacial diffusion in fcc TaN, a $2 \times 2$ supercell in $[11 \overline{2}]$ and $[1 \overline{1} 0]$ directions is used, including three $\mathrm{Cu}$ layers and six TaN layers. The supercell contains $24 \mathrm{Ta}$ s, 24 $\mathrm{N}$, and $24 \mathrm{Cu}$ atoms before a vacancy is introduced. Four diffusion processes are considered with the vacancy placed at either the top or the second layer of the substrate with both $\mathrm{Ta}$ and $\mathrm{N}$ terminations.

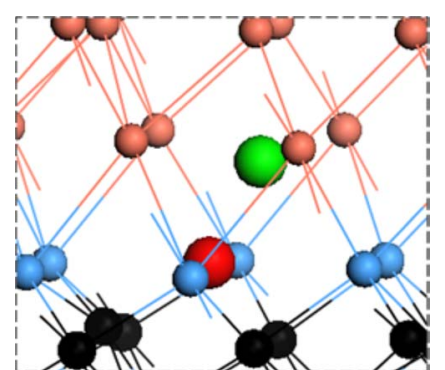

(a)

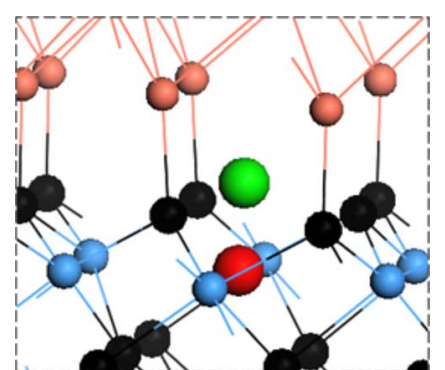

(b)

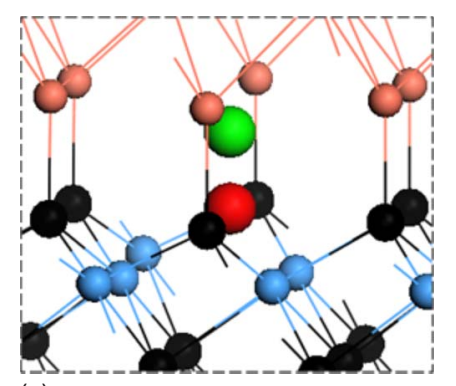

(c)

(d)
The results are rather dramatic: upon relaxation of the final states, the $\mathrm{Cu}$ atom originally occupying the $\mathrm{Ta}$ or $\mathrm{N}$ vacancy is repelled back, indicating that the vacancy sites are not energetically stable for $\mathrm{Cu}$ occupation. Thus no diffusion barrier can be extracted. The only exception is the $\mathrm{N}$ vacancy at the second layer of Ta-terminated substrate. In this case, although a final state can be located (i.e., $\mathrm{Cu}$ is stable in the $\mathrm{N}$ vacancy), its energy is $4.6 \mathrm{eV}$ higher than the initial state. Moreover, the diffusion energy barrier from the initial to final state is a staggering $5.4 \mathrm{eV}$ (Fig. 7). The results strongly suggest that the interfacial diffusion of $\mathrm{Cu}$ into fcc TaN is highly improbable: $\mathrm{Cu}$ is either not stable in a vacancy site or its diffusion barrier is way too high.

To be complete, we have calculated bulk diffusion barriers in fcc TaN following the same procedure outlined for bcc Ta. The supercell consists of $48 \mathrm{Ta}$ and $48 \mathrm{~N}$ atoms, $36 \mathrm{Ta}$ and $36 \mathrm{~N}$ atoms, and $24 \mathrm{Ta}$ and $24 \mathrm{~N}$ atoms for diffusion along [100], [110], and [111] directions, respectively. The results are summarized in Fig. 8. The lowest bulk diffusion barrier is $1.2 \mathrm{eV}$ and the corresponding diffusion paths are along [110] direction via Ta vacancies or along [100] direction via adjacent TaN-Ta vacancies. The bulk diffusion barrier in fcc TaN almost doubles that in bcc Ta, indicating that $\mathrm{Cu}$ atoms will be practically immobile even if they manage to diffuse into fcc TaN across the interface.

Next we discuss briefly the strain effect on the diffusion energy barriers. In all our calculations so far, $\mathrm{Cu}$ layers were stretched to fit the substrate lattice, e.g., $11 \%$ tensile strain on bcc Ta and $21 \%$ tensile strain on fcc TaN, respectively, and the substrate is strain-free. However, as $\mathrm{Cu}$ layers grow thicker, a compression strain could develop in $\mathrm{Cu}$, which reduces the tensile strain of $\mathrm{Cu}$ layers at the interface and introduces a compression in the substrate. To model this strain effect on diffusion, we apply uniform compression strains upon the previous pseudomorphic models. Once

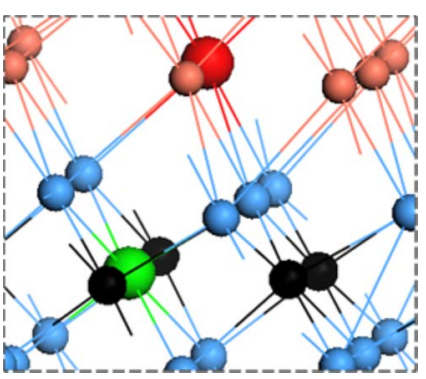

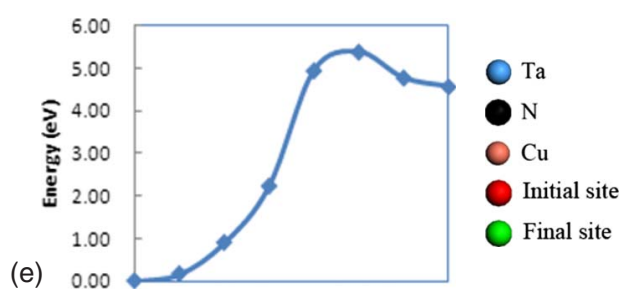

FIG. 7. (Color online) $\mathrm{Cu} / \mathrm{fcc}-\mathrm{TaN}$ interfacial diffusions. No stable lattice site in the substrate can be found for Cu occupation (a) with a Ta vacancy at the interface, (b) with a Ta vacancy at one layer below the interface, or (c) with a $\mathrm{N}$ vacancy at the interface. For all three cases here, the $\mathrm{Cu}$ atom was initially placed at the vacancy (Initial site); after the relaxation, the $\mathrm{Cu}$ atom was "repelled" back and its final position is represented by the green sphere. The only exception is shown in (d) where the final stable site is a $\mathrm{N}$ vacancy at one layer below the interface, represented by the green sphere. The energy profile for the diffusion is shown in (e) with a huge barrier of $5.4 \mathrm{eV}$. 


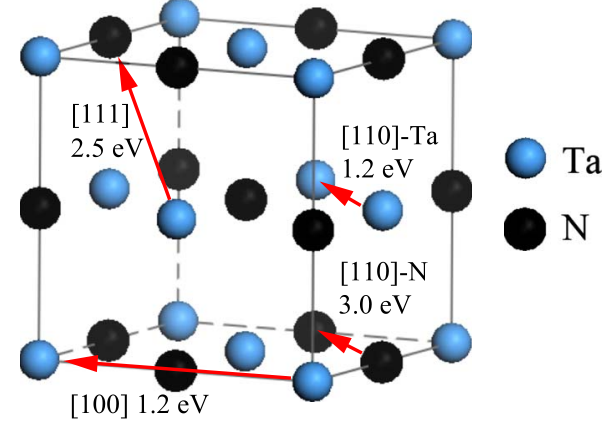

FIG. 8. (Color online) Bulk diffusion barriers of $\mathrm{Cu}$ in fcc TaN are shown in different directions. The arrow points from the diffusing $\mathrm{Cu}$ atom to the vacancy in each direction of interest.

again, pseudomorphic models capture the essential physics taking place at the interface and at the same time render the computation feasible. We found that although the diffusion energy barrier changes as a function of applied strain, the general conclusions remain the same. For example, with a $5 \%$ compression, no stable final configuration was found for $\mathrm{Cu}$ occupying TaN interfacial vacancies-the diffusing $\mathrm{Cu}$ atom was pushed back at the $\mathrm{Cu} / \mathrm{fcc}-\mathrm{TaN}$ interface. As a result, no diffusion barrier can be obtained. For $\mathrm{Cu} / \mathrm{bcc} \mathrm{Ta}$, we found that $\mathrm{Cu}$ diffusion to bcc $\mathrm{Ta}$ has a relatively lower energy barrier (comparing to fcc TaN) of $0.3 \mathrm{eV}$ with $0.4 \mathrm{eV}$ energy drop between the initial and final states. These results are consistent with the general conclusion that $\mathrm{Cu}$ diffusion into bcc $\mathrm{Ta}$ is energetically preferred with relatively lower energy barriers while the interfacial diffusion into fcc $\mathrm{TaN}$ is energetically strongly unfavorable.

One can understand the reason why the interfacial diffusion barrier of fcc TaN is much higher than that of bcc Ta by considering the bulk vacancy formation energy. The vacancy formation energy in $\mathrm{Cu}$ is much lower than that in bec Ta; therefore it is energetically favorable for a $\mathrm{Cu}$ atom to occupy a vacancy in bcc $\mathrm{Ta}$, leaving behind a $\mathrm{Cu}$ vacancy. However, the situation is opposite in fcc TaN: the vacancy formation energy is negative, i.e., Ta vacancies are energetically preferred in fcc TaN; therefore it is unfavorable for $\mathrm{Cu}$ to occupy a Ta vacancy. Since the formation energy of $\mathrm{N}$ vacancy in fcc $\mathrm{TaN}$ is either negative (for Ta rich) or positive but smaller (for $\mathrm{N}$ rich) than that of $\mathrm{Cu}$, it is also energetically unfavorable for $\mathrm{Cu}$ to occupy the $\mathrm{N}$ vacancy in fcc $\mathrm{TaN}$. This result should be contrasted to that in $\mathrm{Cu} / \mathrm{Si}$ interfacial diffusion. Although the vacancy formation in $\mathrm{Si}$ is similar to that in bcc Ta, the physics of $\mathrm{Cu} / \mathrm{Si}$ and $\mathrm{Cu} / \mathrm{Ta}$ interfacial diffusions is very different. Since $\mathrm{Cu}$ chemically reacts with $\mathrm{Si}, \mathrm{Cu}$ diffusion into $\mathrm{Si}$ is primarily driven by the chemical reactions, not controlled by vacancy formation in $\mathrm{Si}$. The fact that $\mathrm{Cu}$ can spontaneously diffuse into $\mathrm{Si}$ vacancy suggests that $\mathrm{Cu}$ atoms indeed prefer to intermix with $\mathrm{Si}$ atoms. On the contrary, $\mathrm{Cu}$ is not believed to react with bcc Ta and no stable Cu-Ta compound has ever been observed. Therefore the intermixing of $\mathrm{Cu}$ and $\mathrm{Ta}$ is entirely accomplished by interfacial diffusion. The large $\mathrm{Cu}$ diffusion barriers observed in bulk Ta reflects the immiscibility of $\mathrm{Cu}$ and Ta. The high value of vacancy formation energy in Ta is the limiting factor for the $\mathrm{Cu}$ diffusion.
One can also make sense of the diffusion barriers from the atomic structure point of view. It is found that the diffusion barrier along different direction is correlated with the corresponding $\mathrm{Cu}-\mathrm{Ta}$ bond length at their saddle points. Highenergy barriers at the saddle point result from large compressions of the bonds. For example, in bcc $\mathrm{Ta}$, the $\mathrm{Cu}-\mathrm{Ta}$ bond lengths at the saddle points for [111], [100], and [110] diffusions are 2.7, 2.5, and $2.3 \AA$, respectively, and they correspond to an energy barrier of $0.65,2.7$, and $5.8 \mathrm{eV}$, respectively, shown in Fig. 8. The bonding environment in fcc TaN is more complicated but the general trend applies as well. The $\mathrm{Cu}-\mathrm{Ta}$ bond lengths at the saddle points for diffusion along [100], [110] N-vacancy, and [111] directions are 2.4, 2.3, and $2.1 \AA$, respectively, corresponding to an energy barrier of $1.2,2.5$, and $3.0 \mathrm{eV}$.

\section{CONCLUSION}

We perform DFT calculations for various interfacial structures, including $\mathrm{Cu} / \mathrm{bcc} \mathrm{Ta}, \mathrm{Cu} / \mathrm{fcc} \mathrm{TaN}, \mathrm{Cu} / \mathrm{hex} \mathrm{Ta}_{2} \mathrm{~N}, \mathrm{Cu} /$ hex $\mathrm{TaN}$, and $\mathrm{Cu} / \mathrm{Si}$. Different surface orientations, in-plane arrangements, and surface terminations are examined in detail. It is found that $\mathrm{Cu} / \mathrm{bcc}-\mathrm{Ta}$ and $\mathrm{Cu} / \mathrm{fcc}-\mathrm{TaN}$ interfaces are most stable from the cohesive energy point of view. The preferred interfacial structures are dictated by the coexistence of strong $\mathrm{Cu}-\mathrm{N}$ ionic bonding and $\mathrm{Cu}-\mathrm{Ta}$ covalent/ metallic bonding.

Based on the results of interfacial structure, we carry out DFT-NEB calculations for diffusion energy barriers across the interfaces, and in bulk of bcc Ta and fcc TaN. As a comparison, the diffusion energy barriers are also determined for $\mathrm{Cu} / \mathrm{Si}$ interfaces. We find that $\mathrm{Cu}$ atom can diffuse spontaneously into a $\mathrm{Si}$ vacancy across $\mathrm{Cu}(111) / \mathrm{Si}$ (111) interface. There is a smaller energy barrier of $0.08 \mathrm{eV}$ for $\mathrm{Cu}$ to cross $\mathrm{Cu}(100) / \mathrm{Si}(100)$ interface. Although the diffusion barrier of $\mathrm{Cu}$ into a Ta vacancy across $\mathrm{Cu}(111) / \mathrm{bcc}-\mathrm{Ta}$ (110) interface is only $0.07 \mathrm{eV}$, the vacancy formation energy of Ta is very high, limiting the rate of interfacial diffusion. Because vacancy formation is energetically preferred in fcc $\mathrm{TaN}, \mathrm{Cu}$ diffusion into fcc-TaN vacancy is nearly impossible, with either no stable lattice sites for $\mathrm{Cu}$ occupation or with extremely high diffusion energy barrier $(5.4 \mathrm{eV})$. Although both bcc Ta and fcc TaN can serve as diffusion barrier materials, their working mechanisms are different: $\mathrm{Cu}$ diffusion into bcc Ta is suppressed due to the high formation energy of $\mathrm{Ta}$ vacancy; $\mathrm{Cu}$ diffusion into fcc $\mathrm{TaN}$ is prohibited because of the extremely high interfacial diffusion energy barrier. Overall, fcc TaN is a better material for diffusion barrier. In this work, we have not considered the diffusions along grain boundaries or dislocations, which could be important. However, such calculations are beyond the scope of firstprinciples simulations and thus should be left for future studies, possibly by multiscale modeling.

\section{ACKNOWLEDGMENTS}

This work was supported by Intel Corp. and NSF under Grant No. DMR-0611562. We thank Sadasivan Shankar for many valuable discussions. 
${ }^{1}$ Y. J. Lee, B. S. Suh, M. S. Kwon, and C. O. Park, J. Appl. Phys. 85, 1927 (1999).

${ }^{2}$ C. K. Hu and J. M. E. Harper, Mater. Chem. Phys. 52, 5 (1998).

${ }^{3}$ A. E. Kaloyeros and E. Eisenbraun, Annu. Rev. Mater. Sci. 30, 363 (2000).

${ }^{4}$ C. E. Ramberg, E. Blanquet, M. Pons, C. Bernard, and R. Madar, Microelectron. Eng. 50, 357 (2000).

${ }^{5}$ H. C. Chung and C. P. Liu, Surf. Coat. Technol. 200, 3122 (2006).

${ }^{6}$ K. Holloway, P. M. Fryer, C. Cabral, Jr., J. M. E. Harper, P. J. Bailey, and K. H. Kelleher, J. Appl. Phys. 71, 5433 (1992).

${ }^{7}$ M. Stavrev, C. Wenzel, A. Möller, and K. Drescher, Appl. Surf. Sci. 91, 257 (1995).

${ }^{8}$ S. P. Chong, Y. C. Ee, Z. Chen, and S. B. Law, Surf. Coat. Technol. 198, 287 (2005).

${ }^{9}$ L. Gladczuk, A. Patel, J. D. Demaree, and M. Sosnowski, Thin Solid Films 476, 295 (2005).

${ }^{10} \mathrm{~W}$. Wu, K. Ou, C. Chou, and C. Wu, J. Electrochem. Soc. 150, G83 (2003).

${ }^{11}$ J. Nazon, B. Fraisse, J. Sarradin, S. G. Fries, J. C. Tedenac, and N. Fréty, Appl. Surf. Sci. 254, 5670 (2008).

${ }^{12}$ D. Kwon, H. Park, S. Ghosh, C. Lee, H. T. Jeon, and J. G. Lee, J. Korean Phys. Soc. 44, 1108 (2004).

${ }^{13}$ J. H. Hsieh, C. M. Wang, and C. Li, Surf. Coat. Technol. 200, 3179 (2006).

${ }^{14}$ H. B. Nie, S. Y. Xu, S. J. Wang, L. P. You, Z. Yang, C. K. Ong, J. Li, and T. Y. F. Liew, Appl. Phys. A: Mater. Sci. Process. 73, 229 (2001).

${ }^{15}$ M. Sahnoun, C. Daul, M. Driz, J. C. Parlebas, and C. Demangeat, Comput. Mater. Sci. 33, 175 (2005).

${ }^{16}$ C. Stampfl and A. J. Freeman, Phys. Rev. B 71, 024111 (2005).

${ }^{17}$ P. Violet, E. Blanquet, and O. Le Bacq, Microelectron. Eng. 83, 2077 (2006).

${ }^{18}$ L. Yu, C. Stampfl, D. Marshall, T. Eshrich, V. Narayanan, J. M. Rowell, N. Newman, and A. J. Freeman, Phys. Rev. B 65 , 245110 (2002).

${ }^{19}$ N. Iwamoto, N. Truong, and E. Lee, Thin Solid Films 469, 431 (2004).

${ }^{20}$ S. W. Loh, D. H. Zhang, C. Y. Li, R. Liu, and A. T. S. Wee, Thin Solid Films 462-463, 240 (2004).

${ }^{21}$ S. Shankar, H. Simka, and M. Haberty, J. Phys.: Condens. Matter 20, 064232 (2008).
${ }^{22}$ W. K. Kuhn, R. A. Campbell, and D. W. Goodman, J. Phys. Chem. 97, 446 (1993).

${ }^{23}$ A. Hashibon, C. Elsässer, Y. Mishin, and P. Gumbsch, Phys. Rev. B 76, 245434 (2007).

${ }^{24}$ L. Chen, N. Magtoto, B. Ekstrom, and J. Kelber, Thin Solid Films 376, 115 (2000).

${ }^{25}$ V. Venugopal, Ph.D. thesis, Technische Universiteit Delft, 2005.

${ }^{26}$ T. P. C. Klaver and B. J. Thijsse, Molecular Dynamics Study of $\mathrm{Cu}$ Thin Film Deposition on $\beta$-Ta, Mat. Res. Soc. Symp. Proc. No. 721 (Materials Research Society, Warrendale PA, 2002), p. 37-42.

${ }^{27}$ R. A. Araujo, J. Yoon, X. H. Zhang, and H. Y. Wang, Thin Solid Films 516, 5103 (2008).

${ }^{28}$ M. H. Tsai, S. C. Sun, C. E. Tsai, S. H. Chuang, and H. T. Chiu, J. Appl. Phys. 79, 6932 (1996).

${ }^{29}$ G. Henkelman, B. P. Uberuaga, and H. Jónsson, J. Chem. Phys. 113, 9901 (2000).

${ }^{30} \mathrm{G}$. Henkelman and H. Jónsson, J. Chem. Phys. 113, 9978 (2000).

${ }^{31}$ G. Kresse and J. Hafner, Phys. Rev. B 49, 14251 (1994).

${ }^{32}$ G. Kresse and J. Furthmüller, Comput. Mater. Sci. 6, 15 (1996).

${ }^{33}$ D. Vanderbilt, Phys. Rev. B 41, 7892 (1990).

${ }^{34}$ J. P. Perdew, in Electronic Structure of Solids '91, edited by P. Ziesche and H. Eschrig (Akademie Verlag, Berlin, 1991).

${ }^{35}$ C. Kittel, Introdunction to Solid State Physics, 5th ed. (Wiley, New York, 1976).

${ }^{36}$ J. K. Solberg, Acta Crystallogr., Sect. A: Cryst. Phys., Diffr., Theor. Gen. Crystallogr. 34, 684 (1978).

${ }^{37}$ F. J. Walker, E. D. Specht, and R. A. McKee, Phys. Rev. Lett. 67, 2818 (1991).

${ }^{38}$ Y. S. Choi, A. K. Petford-Long, R. C. C. Ward, and M. R. Wells, Thin Solid Films 413, 41 (2002).

${ }^{39}$ C. A. Chang, J. Appl. Phys. 67, 566 (1990).

${ }^{40}$ F. Soisson and C. C. Fu, Phys. Rev. B 76, 214102 (2007).

${ }^{41}$ J. P. Ehrhart, P. Jung, H. Schultz, and H. Ullmaier, Atomic Defects in Metals, Landolt-Bönstein New Series, Group III (Springer-Verlag, Berlin, 1991), Vol. 25.

${ }^{42}$ A. F. Wright, Phys. Rev. B 74, 165116 (2006).

${ }^{43}$ P. M. Derlet, D. Nguyen-Manh, and S. L. Dudarev, Phys. Rev. B 76, 054107 (2007).

${ }^{44}$ Z. Alahmed and H. X. Fu, Phys. Rev. B 76, 224101 (2007). 